\title{
The ФBT1 large serine recombinase catalyzes DNA integration at pseudo-attB sites in the genus Nocardia
}

\author{
Marion Herisse $^{1}$, Jessica L Porter ${ }^{1}$, Romain Guerillot ${ }^{1}$, Takehiro Tomita ${ }^{2}$, Anders Goncalves Da Silva ${ }^{1,2}$, \\ Torsten Seemann ${ }^{1,2}$, Benjamin P Howden ${ }^{1,2,3}$, Timothy P Stinear ${ }^{\text {Corresp., } 1,2,3 \text {, Sacha J Pidot }}{ }^{\text {Corresp. } 1}$ \\ 1 Department of Microbiology and Immunology at the Doherty Institute, University of Melbourne, Melbourne, Australia \\ 2 Microbiological Diagnostic Unit, University of Melbourne, Melbourne, Australia \\ 3 Doherty Applied Microbial Genomics, University of Melbourne, Melbourne, Australia \\ Corresponding Authors: Timothy P Stinear, Sacha J Pidot \\ Email address: tstinear@unimelb.edu.au, spidot@unimelb.edu.au
}

Plasmid vectors based on bacteriophage integrases are important tools in molecular microbiology for the introduction of foreign DNA, especially into bacterial species where other systems for genetic manipulation are limited. Site specific integrases catalyze recombination between phage and bacterial attachment sites (attP and att $B$, respectively) and the best studied integrases in the Actinomycetes are the serine integrases from the Streptomyces bacteriophages $\Phi \mathrm{C} 31$ and $\Phi \mathrm{BT} 1$. As this reaction is unidirectional and highly stable, vectors containing phage integrase systems have been used in a number of genetic engineering applications. Plasmids bearing the $\Phi B T 1$ integrase have been used to introduce DNA into Streptomyces and Amycolatopsis strains, however, they have not been widely studied in other actinobacterial genera. Here, we show that vectors based on $\triangle \mathrm{BT} 1$ integrase can stably integrate into the chromosomes of a range of Nocardia species, and that this integration occurs despite the absence of canonical attB sites in these genomes. Furthermore, we show that a ФBT1 integrase-based vector can insert at multiple pseudo-attB sites within a single strain and we determine the sequence of a pseudo-attB motif. These data suggest that $\Phi B T 1$ integrase-based vectors can be used to readily and semi-randomly introduce foreign DNA into the genomes of a range of Nocardia species. However, the precise site of insertion will likely require empirical determination in each species to avoid unexpected off-target effects. 
1 The $\Phi B$ T1 large serine recombinase catalyzes DNA integration at pseudo-attB sites in the

2 genus Nocardia

3

4 Marion Herisse $^{1}$, Jessica L. Porter ${ }^{1}$, Romain Guerillot ${ }^{1}$, Takehiro Tomita ${ }^{2}$,

5 Anders Goncalves Da Silva ${ }^{1,2}$, Torsten Seemann ${ }^{1,2}$, Benjamin P. Howden ${ }^{1,2,3}$, Timothy P.

6 Stinear $^{1,3}$, Sacha J. Pidot ${ }^{1}$

7

81 Department of Microbiology and Immunology at The Doherty Institute, University of

9 Melbourne, Melbourne, Australia, 3000

$10{ }^{2}$ Microbiological Diagnostic Unit, University of Melbourne, Melbourne, Australia, 3000

$11{ }^{3}$ Doherty Applied Microbial Genomics, University of Melbourne, Melbourne, Australia, 3000

12 Joint corresponding authors: Timothy P Stinear and Sacha J Pidot

13

14 Email address: tstinear@unimelb.edu.au, spidot@unimelb.edu.au

15

16 


\section{Abstract:}

Plasmid vectors based on bacteriophage integrases are important tools in molecular microbiology for the introduction of foreign DNA, especially into bacterial species where other systems for genetic manipulation are limited. Site specific integrases catalyze recombination between phage and bacterial attachment sites ( $a t t P$ and $a t t B$, respectively) and the best studied integrases in the Actinomycetes are the serine integrases from the Streptomyces bacteriophages ФC31 and ФBT1. As this reaction is unidirectional and highly stable, vectors containing phage integrase systems have been used in a number of genetic engineering applications. Plasmids bearing the $\Phi B T 1$ integrase have been used to introduce DNA into Streptomyces and Amycolatopsis strains, however, they have not been widely studied in other actinobacterial genera. Here, we show that vectors based on $\Phi$ BT1 integrase can stably integrate into the chromosomes of a range of Nocardia species, and that this integration occurs despite the absence of canonical attB sites in these genomes. Furthermore, we show that a $\Phi B \mathrm{~T} 1$ integrase-based vector can insert at multiple pseudo-attB sites within a single strain and we determine the sequence of a pseudo-attB motif. These data suggest that $\Phi B T 1$ integrase-based vectors can be used to readily and semi-randomly introduce foreign DNA into the genomes of a range of Nocardia species. However, the precise site of insertion will likely require empirical determination in each species to avoid unexpected off-target effects.

\section{Introduction:}

The actinomycetes comprise diverse bacterial genera, ranging from those well known for their prolific production of secondary metabolites (Streptomyces spp.) to those that contain some of world's most successful pathogens (Mycobacterium spp.). Nocardia species are 
41 actinomycetes that have features in common with pathogens and secondary metabolite 42 producers. For example, several species of Nocardia (i.e. Nocardia asteroides, Nocardia 43 brasiliensis) are opportunistic human and animal pathogens, causing lung, skin and brain 44 infections, predominantly in immunocompromised hosts, while others are reservoirs of bioactive natural products (Hoshino et al. 2011; Tanaka et al. 1997) and are capable of degrading or converting widespread environmental pollutants (Hahn et al. 2013; Santillan et al. 2016). These beneficial properties and their potential biotechnical applications have attracted a great deal of interest in the genus Nocardia. However, genetic tools to manipulate Nocardia spp. are limited in comparison to better studied actinomycetes, such as Streptomyces spp.

Serine integrases are widely used tools in the molecular biology of a range of species, especially actinomycetes. These integrase proteins (Int) are phage-encoded enzymes that mediate the site-specific recombination of DNA molecules between two sites (known as "attachment sites", or att-sites) on the phage (attP) and host genome (attB) (Rutherford \& Van Duyne 2014). As excision of the integrated DNA requires a separate protein (recombination directionality factor), the integration reaction is both highly directional and stable (Fogg et al. 2014). It is these two key features that have made these enzymes such attractive tools for the modification of

57 bacterial and mammalian genomes (Stark 2017).

Perhaps the best studied serine integrases are those derived from Streptomyces bacteriophages, including $\Phi \mathrm{C} 31$ and $\Phi \mathrm{BT} 1$. Vectors based on the integrase (int) from $\Phi \mathrm{C} 31$ and ФBT1 have been utilised to insert DNA into a range of genomes in both prokaryotes (including bacteria and archaea) and eukaryotes (yeast, plant and mammalian cells) (Baltz 2012). The attP and $a t t B$ sites for members of the serine recombinase family are often $<50$ bp in length and contain imperfect inverted repeat sequences that surround a core dinucleotide, known as the 
64 crossover site (Smith \& Thorpe 2002). In Streptomyces coelicolor, both ФC31 Int and ФBT1 Int

65 mediate DNA insertion into separate genes (SCO3798, a pirin homologue and SCO4848, a

66 putative membrane protein, respectively), inactivating their targets (Gregory et al. 2003;

67 Kuhstoss et al. 1991). While $\Phi C 31$ attB sites are highly similar in a number of Streptomyces

68 species, non-specific integration in sequences that have partial identity to $a t t B$ (pseudo-attB sites)

69 has also been reported, although this appears to occur relatively infrequently (Baltz 2012;

70 Combes et al. 2002). While vectors utilizing $\Phi B T 1$ Int have also been shown to integrate in a

71 range of Streptomyces species and Amycolatopsis mediterranei, their usage outside these

72 organisms has been limited (Gregory et al. 2003; Li et al. 2017). Furthermore, the extent to which $\Phi B T 1$ Int can catalyse integration at pseudo- $a t t B$ sites is unknown.

Here, we show that $\Phi B T 1$ Int can be used to integrate foreign DNA into a range of

Nocardia species and that these insertions occur despite the absence of a canonical attB site. We

show that these pseudo-attB sites are unique to each species, that certain sites are preferred within certain species and that minimal homology to the canonical attB is required for DNA insertion. Furthermore, using this range of unique pseudo-attB sites we identify a pseudo-attB motif and delineate the nucleotides important for DNA integration.

Methods:

$\underline{\text { Microbial culture }}$

Nocardia strains were routinely cultured in Brain Hear Infusion (BHI) broth or on BHI 84 agar plates (Difco). E. coli strains were cultured in Luria broth or on Luria agar plates. Streptomyces species were cultured on MS agar for conjugation and isolation of transconjugants 86 (Kieser et al. 2000). All strains used in this study are listed in Table S1. The plasmid pRT801, an 
87 E. coli-Streptomyces integrating vector containing the BT1 int gene and attP site, aac3(iv)

88 (encoding apramycin resistance) and a Streptomyces oriT for conjugative transfer, was used in

89 this study and has been previously published (Gregory et al. 2003).

$\underline{\text { DNA extraction procedures }}$

Genomic DNA was extracted from Nocardia strains according to a modified DNA isolation method based on two previously published protocols (Belisle \& Sonnenberg 1998) (Gonzalez-y-Merchand et al. 1996). Briefly, a $10 \mathrm{ml}$ culture of cells was grown in BHI 95 supplemented with $2 \%$ glycine for 4 days at $30{ }^{\circ} \mathrm{C}$ with shaking at $200 \mathrm{rpm}$. Cells were pelleted and resuspended in $2 \mathrm{ml}$ Tris- $\mathrm{HCl} \mathrm{pH} 8.0$ and mixed with an equal volume of 1:1 chloroform: methanol for 10 mins with gentle shaking. Cells were pelleted at 8000 x $g, 10 \mathrm{~min}$, then both the organic and aqueous phases were removed, leaving only the cells. Cells were dried to remove all traces of chloroform and methanol and were resuspended in $475 \mu \mathrm{l}$ of TE buffer, followed by the addition of lysozyme at a final concentration of $5 \mathrm{mg} / \mathrm{ml}$. Cells were incubated for $2 \mathrm{~h}$ at $37{ }^{\circ} \mathrm{C}$ and then pelleted at $8000 \times \mathrm{g}, 10 \mathrm{~min}$ and the supernatant removed. The cell pellet was then resuspended in 500ul lysis buffer (6 M guanidine $\mathrm{HCl}, 10 \mathrm{mM}$ EDTA, $1 \mathrm{mM} \beta$-mercaptoethanol, ice/ethanol bath, followed by thawing at $60{ }^{\circ} \mathrm{C}$. This was repeated three times, then the cell suspension was extracted twice with chloroform. The supernatant was removed to a separate tube and the DNA was precipitated using ethanol. DNA was resuspended in $50 \mu 1$ of $10 \mathrm{mM}$ Tris- $\mathrm{HCl}$ and stored at $-20{ }^{\circ} \mathrm{C}$.

Plasmid DNA was extracted from E. coli DH10B cells containing pRT801 using a 109 plasmid mini-prep kit (Favorgen Biotech). 
112 (Ishikawa et al. 2006). In brief, Nocardia strains were grown in 100ml BHI containing 2\%

113 glycine for 4 days at $30{ }^{\circ} \mathrm{C}$ prior to transformation. Cells were harvested and washed twice with

$11450 \mathrm{ml}$ ice-cold water. The cells were then resuspended in $500 \mu 1$ of ice-cold $10 \%$ glycerol and 50

$115 \mu \mathrm{l}$ was used per transformation and transferred to a chilled electroporation cuvette (2 mm gap).

116 Approximately $\sim 1 \mu \mathrm{g}$ of DNA was added and cells were pulsed at $2.4 \mathrm{kV}$ using an electroporator

117 (Electro Cell Porator 600, BTX Inc.). To each transformation, $900 \mu$ of BHI broth was added 118 and the cells were incubated for $2 \mathrm{~h}$ at $37^{\circ} \mathrm{C} . N$. terpenica, N. uniformis and N. brasiliensis 119 transformations were plated onto BHI plates containing $50 \mu \mathrm{g} / \mathrm{ml}$ of apramycin, while $N$. 120 arthritidis transformations were plated onto BHI plates containing $100 \mu \mathrm{g} / \mathrm{ml}$ apramycin. All 121 plates were incubated for 2-3 days at $30^{\circ} \mathrm{C}$. For each Nocardia strain analysed for pRT801

122 insertions sites, transformants were selected from independent transformation reactions to avoid 123 detecting sibling colonies. As such, transformants were picked from 14 independent 124 transformation reactions for Nocardia terpenica; 6 independent transformations for both 125 Nocardia brasiliensis and Nocardia uniformis; and 5 independent transformations for Nocardia 126 arthritidis.

128 Conjugation of plasmids into Streptomyces strains

DNA was transferred from E. coli ET12567 (pUZ8002) to Streptomyces species by conjugation according to a previously published protocol (Kieser et al. 2000).

132 Confirmation of pRT801 integration in Streptomyces strains 
134 using the following primers: ScBT1-F - CCCAATACGAAGGAGACGAT; ScBT1-R 135 GCTCATTCACAACGACAACG. Insertion of pRT801 in Streptomyces albus was also 136 determined by PCR, but due to differences upstream of the attB site in $S$. albus, a different 137 forward primer (SaBT1-F - GGGGTGGGGTTCTTCTCAC) was used in conjunction with the 138 ScBT1-R primer.

DNA sequencing and analysis

Complete sequencing of the $N$. terpenica genome was performed with a combination of

Pacbio SMRT and Illumina sequence data. N. terpenica DNA was extracted as outlined above and prepared for sequencing on the Pacbio RSII using the Template Prep Kit 1.0 (Pacbio). Genomic DNA was size selected after adapter ligation using a BluePippin system (Sage Biosciences) with a $10 \mathrm{~kb}$ cutoff. Adapter-ligated, circularised DNA was loaded onto a single SMRT cell at $0.2 \mathrm{nM}$ and sequence data were captured with a $6 \mathrm{~h}$ movie time. To complete the genome sequence of $N$. terpenica, Pacbio sequencing data was assembled using HGAP3, as implemented in the SMRT Portal (Pacbio), resulting in a single contig. This contig was polished three times using Quiver (Pacbio) before final indel error correction with Illumina reads using Snippy v3.2 (https://github.com/tseemann/snippy).

152 preparation kit (Illumina) and whole genome sequencing was performed on the NextSeq 153 platform (Illumina) with $2 \times 150$ bp paired-end chemistry. A sequencing depth of $>50 \times$ was 154 targeted for each sample. Samples with Illumina only data were assembled with SPAdes (v 155 3.10.1) (Bankevich et al. 2012) and annotated with Prokka v 1.12 (Seemann 2014). Sequencing 
156 reads are available under BioProject ID PRJNA433999. Table S2 lists each read set deposited in

157 SRA, along with species name and corresponding attB site, as listed in Figure S1 and Table S3

158 of the manuscript. The sequence of pRT801 has been deposited in GenBank under accession

159 number MH192349.

$160 \quad$ Insertion sites of pRT801 were manually inspected and identified using Geneious v9.1.6.

161 Motif analysis was performed using MEME (www.memesuite.org, (Bailey \& Elkan 1994)).

162 Rarefaction analysis was performed in R v3.3.2 (R Development Core Team 2016) to estimate

163 the total number of pseudo-attB sites present in the $N$. terpenica genome. To identify sites with

164 homology to the predicted pseudo-attB motif in Nocardia genomes, FIMO was used

165 (http://meme-suite.org/tools/fimo, (Grant et al. 2011)) with a threshold value of $1 e^{-08}$.

166

167 Construction of $r p l$-based phylogenetic tree

168 Sequences of $r p l$ genes ( $r p l B, r p l J, r p l M, r p l S$ and $r p l T$ ) were extracted from 68 Nocardia

169 genome sequences present in GenBank, as well as the four sequenced Nocardia genomes from

170 this study, using the Perl script gene-puller.pl (https://github.com/kwongj/gene-puller). The rpl

171 sequences were manually curated to a consistent length and then concatenated to create a single

1722352 bp pseudo-sequence for each strain and aligned using Clustal W 2.1 (Larkin et al. 2007). A

173 maximum likelihood phylogenetic tree was inferred with IQ-Tree under a GTR+F+I+G4

174 nucleotide substitution model using the alignment of concatenated sequences mentioned above.

175 Branch support for the phylogeny was assessed with 1000 bootstrap replicates (Hoang et al.

176 2018; Nguyen et al. 2015).

177

178 Results 
179

180

181

182

183

184

185

186

187

188

189

190

191

192

193

194

195

196

197

198

199

200

Sequencing of Nocardia terpenica AUSMDU00012715

As part of a separate natural product discovery project we sought to assess the genetic tractability of a human pathogenic Nocardia sp (strain ID: AUSMDU00012715). To first establish the species identity of the strain and provide a solid reference for subsequent molecular investigations, we subjected this isolate to combined PacBio SMRT and Illumina sequencing to produce a single $9,306,871 \mathrm{bp}$ circular chromosome with 8241 predicted CDS and 70 tRNA regions (Figure 1). No plasmids were detected. Subsequent phylogenetic analysis by comparing five $r p l$ gene sequences from this Nocardia isolate and 68 other Nocardia species (including three others sequenced as part of this study; Table S1, S3) showed that this isolate was most closely related to a previously sequenced Nocardia terpenica isolate (14 pairwise SNP differences among a total of 950 potential variable positions), suggesting that it was a member of the species $N$. terpenica (Figure 1).

\section{pRT801 integrates into the Nocardia terpenica genome}

To assess the potential of $N$. terpenica AUSMDU00012715to accept foreign DNA, we began by transforming this isolate using electroporation with a range of plasmids that have been successfully used in Nocardia and other Actinobacteria. These plasmids included a range of replicating as well as integrating vectors. The replicative plasmids pNV19 and pNV119 (Chiba et al. 2007) were competently hosted by $N$. terpenica AUSMDU00012715, as was the ФBT1 Int expressing vector pRT801 (Gregory et al. 2003). However, transformation with other integrating vectors, including those utilizing the $\Phi \mathrm{C} 31$ and $\Phi \mathrm{L} 5$ integrases failed to produce colonies on selective media. 

media, we screened colonies by PCR for the aac(3)iv gene to confirm the presence of pRT801. As a ФBT1 integrase system has not previously been reported to function in Nocardia species, we were unsure where pRT801 may have inserted in the genome of $N$. terpenica transformants. in these transformants. Surprisingly, an analysis of these genomes showed that pRT801 was inserted at a different chromosome location in each transformant, and not at a single unique $a t t B$ site, as observed in Streptomyces species (Gregory et al. 2003). Furthermore, these insertion sites were spread across the genome and had only limited homology to the canonical 73 bp attB site for $\Phi B T 1$ (Gregory et al. 2003), the previously described minimal 36 bp attB site (Zhang et al. 2008), or even to the reported 9 bp common $a t t P$-attB sequence (Gregory et al. 2003). An in silico scan of the $N$. terpenica genome based on these sequenced transformants did not find the canonical Streptomyces coelicolor $\Phi B \mathrm{BT}$ attB site within the genome, suggesting that all insertions in the $N$. terpenica genome were at pseudo-attB sites.

All pRT801 insertion sites in N. terpenica occur at pseudo-attB sites

Although insertion into pseudo- $a t t B$ sites has previously been observed in some 300 times lower than a true $a t t B$ site (Combes et al. 2002). Furthermore, pseudo-attB sites have not been reported for $\Phi B \mathrm{~B} 1$ Int-based vectors. To characterize the extent and nature of these 
224 pseudo-attB sites within the $N$. terpenica genome (Figure 2A). Of the 27 identified pseudo-attB 225 sites, two sites had four integration events each (Nt-3 and Nt-17), while a further two sites had

226

227

228

229

230

231

232

233

234

235

236

237

238

239

240

241

242

243

244

245

246 two insertions each (Nt-6 and Nt-10) and the remaining fifteen different sites had only a single integration event. The identified sites that had multiple integration events occurred in independent pRT801 transformations, meaning that these were not siblings, but rather that some pseudo- $a t t B$ sites are preferred over others in the $N$. terpenica genome.

Analysis of the 27 insertion sites confirmed our initial observations and showed that these pseudo- $a t t B$ sites were scattered across the chromosome (Figure $2 \mathrm{~A}$ ), with insertions in a range of genes and non-coding regions (Table 1). Furthermore, we observed that even though recombination target site precision was $100 \%$, with no deletions or insertions, these sites had only limited homology to the canonical $\Phi B T 1$ attB site (Gregory et al. 2003) (Figure 2B). Indeed, a comparison between all $N$. terpenica pRT801 insertion sites and the previously described $\Phi B T 1$ attB site showed that the only sequence that was completely conserved was a core GT dinucleotide (Figure 2B, Figure S1). A comparison of the N. terpenica pseudo-attB sites with the canonical $73 \mathrm{bp} a t t B$ site revealed that nucleotide identity among these sites was between $27 \%$ and $48 \%$ (Table 1). Comparison with the minimal 36 bp attB site increased the percentage identity somewhat, however, it was still below $60 \%$ for all analysed sites (Table 1 ). This suggests that, even though these pseudo- $a t t B$ sites differ from the reported $S$. coelicolor attB by greater than $40 \%$ (a minimum of 14 out of 36 non-matching nucleotides), ФBT1 Int is still capable of recognising these sites and integrating donor DNA. We also noticed that the insertion sites that had more than one integration event were not the sites with the highest levels of similarity to the canonical $73 \mathrm{bp}$ attB-site or even the $36 \mathrm{bp}$ minimal attB site (Table 1). It appeared that greater nucleotide identity over the minimal attB region correlated with greater 
247 identity over the whole $a t t B$ sequence, but that this did not necessarily correlate with greater

248 identity over the attB-attP site. These observations indicate that the "site-specific" nature of

249 ФBT1 integration events are not dependent on strict adherence to the canonical attB DNA

250 sequence, but rather are tolerant to alterations within this sequence and suggest that DNA

251 topological factors (spacing between certain nucleotides) may also be important.

252

253

254

255

256

257

258

259

260

261

262

263

264

265

266

267

268

269

Due to the diversity of the pseudo-attB sites identified in $N$. terpenica and the fact that some sites were identified more frequently than others, we sought to predict how many possible pseudo- $a t t B$ sites were present in the genome. Rarefaction analysis was performed, which projected a total of 40 insertion sites in the $N$. terpenica genome (Figure 2C), based on those already sequenced. This analysis suggests that there are many more potential insertion sites in the $N$. terpenica genome.

\section{$\underline{\text { Identification of pseudo-attB sites in other Nocardia species }}$}

To confirm whether this phenomenon of pseudo-attB site insertion was seen in other Nocardia species, we transformed pRT801 into three other clinical Nocardia isolates. As for N. terpenica, apramycin resistant colonies of Nocardia brasiliensis, Nocardia arthritidis and Nocardia uniformis were obtained and the presence of aac(3)iv in each transformant was confirmed by PCR. The genomes of several transformants from each species were sequenced, which showed, similarly to $N$. terpenica, that pRT801 had inserted at pseudo-attB sites in all three organisms. Furthermore, none of the pseudo-attB insertion sites in $N$. uniformis, $N$. arthritidis or $N$. brasiliensis were the same as those seen in $N$. terpenica and none of the insertion sites were conserved across the different species. 
272 from 33-52\% when compared to the minimal attB site (Table S3). Likewise, a similar situation

273 was seen for the nine sequenced $N$. arthritidis AUSMDU00012717 isolates, where five unique insertion sites identified in these strains (Table S3, Figure S1) suggests that further pseudo-attB sites remain to be identified. Again, as for $N$. terpenica, we did not find a correlation between sites with the highest identity to $a t t B$ and an increased number of insertions. Interestingly, although the identity between the 9 bp attB-attP overlap site ranged from 4-6 bp, it appears that a minimum 2 bp overlap at this site (Nb-6 (Figure $\mathrm{S} 1$ ), where only the essential GT dinucleotide was conserved) is all that is required for insertion.

Analysis of the 14 sequenced N. uniformis AUSMDU00012718 transformants showed a slightly different pattern, where only two unique insertion sites were identified. The preferred site, found in 13 of the 14 transformants, which we have labelled $\mathrm{Nu}-1$, had 35 identical nucleotides (48\%) to the canonical attB sequence and 22 nucleotides (61\%) that were identical to the minimal $\Phi B T 1$ attB sequence (Table S3, Figure S1). The other pseudo-attB site in this species (named $\mathrm{Nu}-2$ ) was more dissimilar to both the canonical and minimal attB sites than $\mathrm{Nu}-$ 
293 preferred insertion site in N. uniformis. However, given that the level of identity between Nu-1

294 and the minimal $a t t B$ is still only $61 \%$ (14 of 36 non-identical nucleotides), this suggests that

295 increasing identity of pseudo-attB with the canonical attB results in preferential insertion into

296 these sites, although this is not absolute. As for $N$. terpenica, we observed that insertion at each

297 target site occurred with $100 \%$ precision, and confirmed the formation of pseudo-attL and

298 pseudo-attR sites at both the left and right hand ends of each insertion, indicating that these

299 insertions occurred at genuine pseudo-attB sites and were not the result of DNA repair 300 mechanisms (Figure S2).

301

302 Integration of pRT801 plasmid in the genome of Streptomyces species.

303 Non-specific integration at pseudo-attB sites has previously been reported in 304 Streptomyces species for $\Phi C 31$ Int (Baltz 2012; Combes et al. 2002). Sequencing of our 305 Nocardia transformants allowed us to investigate individual insertion sites without any a priori 306 knowledge of their location. Previous studies using ФBT1 Int in Actinomycetes have determined 307 insertion only by PCR around the known attB site (Gregory et al. 2003; Li et al. 2017), meaning 308 that insertions at pseudo-attB sites would have been classed as "non-integrants" and most likely ignored. We sought to determine whether $\Phi$ BT1 pseudo-attB sites exist in various Streptomyces 310 species and if these sites are utilised in strains with canonical attB sites.

312 genomes available in Genbank. We chose species that had previously been shown to be 313 competent for the insertion of plasmid vectors based on ФBT1 Int (Baltz 2012; Gregory et al. 314 2003). A comparison of these $a t t B$ sites showed that they range from $74-100 \%$ identity with $S$. 315 coelicolor attB (Table S4, Figure S3). Variations in these sequences exist within the minimal 
$316 a t t B$ region, as well as within the essential $9 \mathrm{bp}$ core site, confirming our Nocardia findings in

317 which it appears that only partial identity between attP and attB at this crossover site is sufficient 318 for integration.

319 To investigate whether pseudo-attB insertion sites could be identified in Streptomyces 320 species, we introduced pRT801 into S. coelicolor, Streptomyces lividans, Streptomyces albus and 321 Streptomyces cinnamonensis by conjugation. As the genome sequences for $S$. coelicolor, $S$. 322 lividans and S. albus are available, we designed PCR primers surrounding the attB insertion sites 323 in these genomes, where a PCR product would confirm insertion of pRT801 at the canonical attB 324 site. As the $S$. albus ФBT1 attB site has $82 \%$ DNA identity to attB from $S$. coelicolor, we 325 hypothesised that off-target insertions may be more likely in $S$. albus. Fifty $S$. coelicolor, $S$. 326 lividans and S. albus transconjugant colonies were screened by PCR and all were positive for 327 insertion of pRT801 into the canonical attB site (data not shown), indicating that if pseudo-attB 328 site insertions do occur in these species, they are rare events. To confirm that integration had 329 occurred in a single copy and that there were not alterations to the insertion site, we sequenced 330 the genomes of a $S$. coelicolor and a $S$. albus transconjugant. As the genome sequence for $S$. 331 cinnamonensis was unavailable, we initially sequenced four apramycin resistant transconjugants 332 to identify the $a t t B$ site in this species. Integration occurred at the same site in the four sequenced 333 strains and alignment of the $S$. cinnamonensis $\Phi$ BT1 minimal attB with that from $S$. coelicolor 334 showed $91 \%$ identity, with a $100 \%$ overlap in the 9 bp core sequence (Figure 3 ). This suggests 335 that while $\Phi B T 1$ Int can insert DNA at the same genomic region in these various Streptomyces 336 strains, Int has a certain level of tolerance to mutations within attB and also suggests that 337 sequence length and topological factors may be more important than nucleotide identity for DNA 338 integration. 
Delineating the $\Phi$ BT1 pseudo-attB site motif

342 To determine if there was a conserved DNA signature to each of the pseudo-attB sites, motif analysis using MEME was performed. The $36 \mathrm{bp}$ minimal attB regions from all unique sites of the pRT801-containing Nocardia transformants (36 sites total) were analysed. The only motif found to be completely conserved across all sequences was the crossover site, or core GT dinucleotide (Figure 4). However, a 34 bp motif, centred on the core GT dinucleotide, was identified and appears to contain a weakly conserved inverted repeat sequence (Figure 4A). MEME analysis showed the importance of an almost invariant $T$ at position 6 of the motif, as well as highly conserved nucleotides at positions 1, 5, 29, 30 and 34 of the motif (Figure 4A). These conserved positions include TTC at positions 5-7, which is mirrored by GAA at positions 28-30 and both are the same distance from the core GT dinucleotide (11 bases distal on either side) (Figure 4A). These positions lie within the putative zinc ribbon binding domain region of ФBT1 attB (Rutherford et al. 2013) and a number of corresponding bases in attP can be matched within each half site (Figure 4B), showing the bases sufficient for recombination between attP and pseudo- $a t t B$ sites. When examining the presence of the conserved motif nucleotides within the Streptomyces attB and Nocardia pseudo-attB sites, there is partial conservation of the motif within every site (Figure 4A, Figure S4). The sequences containing the closest match to the pseudo- $a t t B$ motif are those from the Streptomycetes, perhaps explaining why $\Phi$ BT1 integrasebased insertions at pseudo-attB sites are not seen in these strains. Furthermore, these data suggest that the presence of an imperfect inverted repeat of approximately the same length as the 
361 minimal attB sequence, surrounding a GT dinucleotide, is sufficient for $\Phi$ BT1 Int catalyzed

362 DNA integration (Figure 4).

363 To confirm our motif prediction, we performed a search for the pseudo-attB motif in the

364 N. terpenica AUSMDU00012715 genome using FIMO (Grant et al. 2011). Fifty-two sites with

365 significant homology to the combined Nocardia pseudo-attB motif were identified at the 366 relatively strict cut-off of $1 e^{-08}$, which is in agreement with the potential number of sites 367 estimated from our rarefaction analysis (Figure 2C). A comparison of these sites identified by 368 FIMO with those identified from the sequenced transformants found four matching sites. This 369 suggests that there may still be additional refinements that can be made to the motif to further match previously identified pseudo-attB sites.

\section{Discussion}

As serine recombinase integration systems lead to unidirectional and highly stable integration of foreign DNA, these genetic tools have been widely used in both prokaryotes and eukaryotes for genetic engineering (Stark 2017). ФBT1 Int has been shown to integrate DNA site-specifically into a single site within $S C O 4848$ (encoding a membrane protein) in the $S$. coelicolor genome and the corresponding attB-site has been mapped (Gregory et al. 2003). ФBT1 Int has also been shown to integrate DNA into an orthologous gene in other Streptomyces species, such as S. albus and S. lividans (Gregory et al. 2003). However, we have shown here that the nucleotide sequences of the $a t t B$-sites in these strains are not identical to the $S$. coelicolor $a t t B$ sequence, revealing that the "site-specific" nature of insertion is tolerant to alterations at various positions within $a t t B$. Here we have shown that $\Phi B T 1$ Int can mediate DNA insertion 
384 into the genomes of several Nocardia species and that insertion occurs at pseudo-attB sites that

385 have as little as $27 \%$ identity to the canonical $a t t B$ site. Furthermore, our use of whole genome

386 sequencing to rapidly identify and confirm the insertion site sequences meant that we were able

387 to avoid the tedious digestion, religation and selection of colonies required for plasmid rescue

388 followed by Sanger sequencing of insertion sites. We propose that for similar studies looking at

389 pseudo-insertion sites of integrases, sequencing costs are no longer prohibitive and allow for the

390 use of whole genome sequencing to investigate such phenomena.

391

The integration sites for integrases from $\Phi C 31$ and $\Phi B T 1$ have been well characterized in

392

393

394

395

396

397

398
Streptomyces species. Previous studies have shown that $\Phi C 31$ Int can catalyze DNA insertion at non- $a t t B$ sequences in $S$. coelicolor in the absence of $a t t B$ (Combes et al. 2002) and that pseudo$a t t B$ sites also exist in the human genome (Thyagarajan et al. 2001). However, pseudo-attB insertion sites for $\Phi B T 1$ Int have not been previously identified. Our data here provide the first description of $\Phi B T 1$ Int insertion of foreign DNA at pseudo-attB sites and show that insertions can occur at multiple pseudo-attB sites within each Nocardia genome. We have also shown that the essential requirement for insertion appears to be the core GT dinucleotide surrounded by an imperfect inverted repeat sequence (Figure 4). A previous study has shown that matching core dinucleotides between $a t t P$ and $a t t B$ of $\Phi B T 1$ are essential for recombination (Zhang et al. 2010). However, our data suggests that multiple substitutions within the minimal 36 bp attB site also do not impact on the ability for recombination to occur, suggesting that the requirements for integration in terms of sequence homology are relatively relaxed, provided the core GT dinucleotide is present.

A comparison of $a t t B$ and Nocardia pseudo-attB sites revealed DNA identities in the range of 27 to $46 \%$ (20 to 34 nucleotides) (Table 1, Table S3). This low level of identity (at most 
407 no better than one in two bases) is also reflected in identity with the minimal attB site, which is

408 at similarly low levels. However, this lack of identity does not seem to inhibit integration, as

409 some of these pseudo-attB sites were identified in multiple transformants from independent

410 transformation reactions. Interestingly, it was not always the sites with the highest identity to $S$.

411 coelicolor attB that were seen most often, but those integration sites that were seen most often do

412 appear to have good conservation of bases that are involved in binding of the zinc ribbon domain

413 (ZD) of ФВТ1 Int and of nucleotides that are putatively involved in binding of the recombinase

414 domain (Figure 4B).

Indeed, based on our deduced motif, certain nucleotides appear to be more important than others for DNA integration, which has also been noted with other large serine recombinases

417 (Rutherford \& Van Duyne 2014). A previous study on the ФC31 integrase showed that certain 418 nucleotides within the minimal $\Phi C 31-a t t B$ sequence are crucial for substrate recognition and 419 interactions between the integrase and its attachment site (i.e. nucleotides $-/+15$ and $-/+16$ ), but 420 also for the efficacy of recombination (i.e. nucleotides -/+2) (Gupta et al. 2007). Interestingly, we 421 found that across all the pseudo- $a t t B$ sites, nucleotides at positions 1 and $34(-/+16$ from the core 422 GT) are relatively well conserved, as are the nucleotides at positions 5 and 30 and 6 and 29 ($423 /+11$ and $-/+12$, respectively) (Figure 4A), which lie within the putative ZD binding region of ФBT1 attB (Rutherford et al. 2013). Indeed, conservation of these sites aligns with the known mutation-sensitive bases present in the $a t t B$ sites of other large serine recombinases, such as ФC31 Int, which occur at similar locations from the core dinucleotide and appear to lie within 427 the ZD and RD binding regions of these integrases (Rutherford et al. 2013; Smith 2015). On the 428 other hand, nucleotides at positions 11 and $24(-/+6), 8$ and $27(-/+9)$ and 2 and $33(-/+15)$ appear 429 to have a random distribution and despite these sites being part of the putative RD and ZD 
430 binding regions, $\Phi B$ T1 Int binding appears to be relatively insensitive to substitutions at these

431 positions . Furthermore, nucleotides at 16 and $19(-/+1)$, and 14 and $21(-/+3)$ are weakly

432 conserved, which correlates well with previous data showing that mutations at these positions in

433 the $S$. coelicolor $\Phi B T 1$ attB site do not affect integration (Zhang et al. 2010). However, a

434 comparison of position $-/+3$ in $a t t P$ and the most commonly observed pseudo-attB sites (Figure

435 4B), shows that these positions correspond well to one another in each half site, suggesting that

436 matching nucleotides at these positions may be part of the reason that these sites were preferred

437 over others. As with the canonical $a t t B$ sequence, the pseudo- $a t t B$ motif identified here also

438 exhibits a weakly conserved inverted repeat centered on the core GT dinucleotide. As mentioned

439 above, the most conserved bases appear between positions $-/+11$ to $-/+16$ from the core,

440 representing approximately $1-1.5$ helical turns of the DNA; which fits well with the $a t t P-a t t B$-Int

441 binding model that has been previously proposed for phage A118 integrase (Rutherford \& Van

442 Duyne 2014; Rutherford et al. 2013).

The identification of four sequenced pseudo-attB sites out of 52 predicted sites, using our motif as a search query, suggests that there are still additional refinements to be made to the motif. Indeed, as an equal number of mutants were not identified in each species, the contribution of sites from each to the motif is unequal, meaning that DNA compositional effects

447 may have biased our motif sequence. It is possible that production of multiple motifs with each

448 based on the pseudo- $a t t B$ sequences obtained from an individual species may lead to more

449 accurate prediction of putative pseudo- $a t t B$ sites within each genome. Further work is required

450 here to understand in detail the impact of changes to individual nucleotides within the $\Phi B T 1$ attB 451 sequence. 

transformability of rare actinomycetes and that ФBT1 Int-catalyzed DNA integration can occur

454 at a number of pseudo sites. This study furthers our knowledge on the integration of serine

455 recombinases and shows that the absence of a specific attB-site does not preclude integration of

456 the phage. Given the potential number of diverse insertion sites in a non-Streptomyces genome,

457 we suggest that caution should be used in interpreting the sites of phage integrase insertions in 458 previously undescribed species.

\section{Conclusion} species and are important tools in these organisms. Here, we have shown that ФBT1 Int based vectors can be transformed into and can integrate stably in a range of Nocardia species. However, integration of pRT801 occurs at pseudo-attB sites in these organisms, and there are multiple pseudo-attB sites in each of these species. An in-depth analysis of these sites showed insertion, although the core GT dinucleotide is essential. Furthermore, the delineation of a pseudo- $a t t B$ motif has revealed those sites that are important for insertion and provides further information on the nucleotides that are important for recognition and integration by large serine 470 recombinases.

\section{References}


473

474

475

476

477

478

479

480

481

482

483

484

485

486

487

488

489

490

491

492

493

494

495

496

497

498

499

500

501

502

503

504

505

506

507

508

509

510

511

512

513

514

515

516

517

518

Bailey TL, and Elkan C. 1994. Fitting a mixture model by expectation maximization to discover motifs in biopolymers. Proceedings of the Second International Conference on Intelligent Systems for Molecular Biology. Menlo Park, California: AAAI Press. p 28-36.

Baltz RH. 2012. Streptomyces temperate bacteriophage integration systems for stable genetic engineering of actinomycetes (and other organisms). Journal of Industrial Microbiology and Biotechnology 39:661-672. 10.1007/s10295-011-1069-6

Bankevich A, Nurk S, Antipov D, Gurevich AA, Dvorkin M, Kulikov AS, Lesin VM, Nikolenko SI, Pham S, Prjibelski AD, Pyshkin AV, Sirotkin AV, Vyahhi N, Tesler G, Alekseyev MA, and Pevzner PA. 2012. SPAdes: a new genome assembly algorithm and its applications to single-cell sequencing. Journal of Computational Biology 19:455-477. 10.1089/cmb.2012.0021

Belisle JT, and Sonnenberg MG. 1998. Isolation of genomic DNA from mycobacteria. Methods Mol Biol 101:31-44. 10.1385/0-89603-471-2:31

Chiba K, Hoshino Y, Ishino K, Takahisa K, Mikami Y, Uehara Y, and Ishikawa J. 2007. Construction of a pair of practical Nocardia-E.coli shuttle vectors. Japanese Journal of Infectious Diseases 60:45-47.

Combes P, Till R, Bee S, and Smith MCM. 2002. The Streptomyces Genome Contains Multiple Pseudo-attB Sites for the C31-Encoded Site-Specific Recombination System. Journal of Bacteriology 184:5746-5752. 10.1128/jb.184.20.5746-5752.2002

Fogg PC, Colloms S, Rosser S, Stark M, and Smith MC. 2014. New applications for phage integrases. J Mol Biol 426:2703-2716. 10.1016/j.jmb.2014.05.014

Gonzalez-y-Merchand JA, Estrada-Garcia I, Colston MJ, and Cox RA. 1996. A novel method for the isolation of mycobacterial DNA. FEMS Microbiology Letters 135:71-77.

Grant CE, Bailey TL, and Noble WS. 2011. FIMO: scanning for occurrences of a given motif. Bioinformatics 27:1017-1018. 10.1093/bioinformatics/btr064

Gregory MA, Till R, and Smith MCM. 2003. Integration Site for Streptomyces Phage BT1 and Development of Site-Specific Integrating Vectors. Journal of Bacteriology 185:53205323. 10.1128/JB.185.17.5320-5323.2003

Gupta M, Till R, and Smith MC. 2007. Sequences in attB that affect the ability of phiC31 integrase to synapse and to activate DNA cleavage. Nucleic Acids Research 35:34073419. 10.1093/nar/gkm206

Hahn V, Sunwoldt K, Mikolasch A, and Schauer F. 2013. Biotransformation of 4-secbutylphenol by Gram-positive bacteria of the genera Mycobacterium and Nocardia including modifications on the alkyl chain and the hydroxyl group. Applied Microbiology and Biotechnology 97:8329-8339. 10.1007/s00253-013-5127-z

Hoang DT, Chernomor O, von Haeseler A, Minh BQ, and Vinh LS. 2018. UFBoot2: Improving the Ultrafast Bootstrap Approximation. Molecular Biology and Evolution 35:518-522. $10.1093 / \mathrm{molbev} / \mathrm{msx} 281$

Hoshino Y, Chiba K, Ishino K, Fukai T, Igarashi Y, Yazawa K, Mikami Y, and Ishikawa J. 2011. Identification of Nocobactin NA Biosynthetic Gene Clusters in Nocardia farcinica. Journal of Bacteriology 193:441-448.

Ishikawa J, Chiba K, Kurita H, and Satoh H. 2006. Contribution of rpoB2 RNA polymerase beta subunit gene to rifampin resistance in Nocardia species. Antimicrobial Agents and Chemotherapy 50:1342-1346. 10.1128/AAC.50.4.1342-1346.2006

Kieser T, Bibb MJ, Buttner MJ, Chater KF, and Hopwood DA. 2000. Practical Streptomyces Genetics: The John Innes Foundation. 
519 Kuhstoss S, Richardson MA, and Rao RN. 1991. Plasmid cloning vectors that integrate site-

520

521

522

523

524

525

526

527

528

529

530

531

532

533

534

535

536

537

538

539

540

541

542

543

544

545

546

547

548

549

550

551

552

553

554

555

556

557

558

559

560

561

562

563 specifically in Streptomyces spp. Gene 97:143-146.

Larkin MA, Blackshields G, Brown NP, Chenna R, McGettigan PA, McWilliam H, Valentin F, Wallace IM, Wilm A, Lopez R, Thompson JD, Gibson TJ, and Higgins DG. 2007. Clustal W and Clustal $\mathrm{X}$ version 2.0. Bioinformatics 23:2947-2948. 10.1093/bioinformatics/btm404

Li C, Zhou L, Wang Y, Zhao G, and Ding X. 2017. Conjugation of varphiBT1-derived integrative plasmid pDZL802 in Amycolatopsis mediterranei U32. Bioengineered:1-6. 10.1080/21655979.2016.1270808

Matsushima P, and Baltz RH. 1996. A gene cloning system for 'Streptomyces toyocaensis'. Microbiology 142 ( Pt 2):261-267. 10.1099/13500872-142-2-261

Murry J, Sassetti CM, Moreira J, Lane J, and Rubin EJ. 2005. A new site-specific integration system for mycobacteria. Tuberculosis (Edinb) 85:317-323. 10.1016/j.tube.2005.08.016

Nguyen LT, Schmidt HA, von Haeseler A, and Minh BQ. 2015. IQ-TREE: a fast and effective stochastic algorithm for estimating maximum-likelihood phylogenies. Molecular Biology and Evolution 32:268-274. 10.1093/molbev/msu300

R Development Core Team. 2016. R: A language and environment for statistical computing. R Foundation for Statistical Computing, Vienna, Austria. URL: http://www.R-project.org/.

Rutherford K, and Van Duyne GD. 2014. The ins and outs of serine integrase site-specific recombination. Current Opinion in Structural Biology 24:125-131. 10.1016/j.sbi.2014.01.003

Rutherford K, Yuan P, Perry K, Sharp R, and Van Duyne GD. 2013. Attachment site recognition and regulation of directionality by the serine integrases. Nucleic Acids Res 41:8341-8356. 10.1093/nar/gkt580

Santillan JY, Dettorre LA, Lewkowicz ES, and Iribarren AM. 2016. New and highly active microbial phosphotriesterase sources. FEMS Microbiology Letters 363. 10.1093/femsle/fnw276

Seemann T. 2014. Prokka: rapid prokaryotic genome annotation. Bioinformatics 30:2068-2069. 10.1093/bioinformatics/btu153

Smith MC. 2015. Phage-encoded Serine Integrases and Other Large Serine Recombinases. Microbiol Spectr 3. 10.1128/microbiolspec.MDNA3-0059-2014

Smith MC, and Thorpe HM. 2002. Diversity in the serine recombinases. Molecular Microbiology 44:299-307.

Stark WM. 2017. Making serine integrases work for us. Current Opinion in Microbiology 38:130-136. 10.1016/j.mib.2017.04.006

Tanaka Y, Hisayuki K, Yazawa K, Mikami Y, Nemoto A, Tojyo T, Kadowaki K, Shigemori H, and Kobayashi J. 1997. Brasilinolide A, a New Macrolide Antibiotic Produced by Nocardia brasiliensis: Producing Strain, Isolation and Biological Activity. Journal of Antibiotics 50:1036-1041.

Thyagarajan B, Olivares EC, Hollis RP, Ginsburg DS, and Calos MP. 2001. Site-specific genomic integration in mammalian cells mediated by phage phiC31 integrase. Molecular and Cellular Biology 21:3926-3934. 10.1128/MCB.21.12.3926-3934.2001

Zhang L, Ou X, Zhao G, and Ding X. 2008. Highly efficient in vitro site-specific recombination system based on streptomyces phage phiBT1 integrase. Journal of Bacteriology 190:6392-6397. 10.1128/JB.00777-08 
564 Zhang L, Wang L, Wang J, Ou X, Zhao G, and Ding X. 2010. DNA cleavage is independent of 565 synapsis during Streptomyces phage phiBT1 integrase-mediated site-specific 566 recombination. Journal of Molecular Cell Biology 2:264-275. 10.1093/jmcb/mjq025

567

568

569 


\section{Table $\mathbf{1}$ (on next page)}

Analysis of pRT801 insertion sites in the $N$. terpenica genome and comparison with the known attB site. 
1 Table 1. Analysis of pRT801 insertion sites in the $N$. terpenica genome and comparison with the 2 known $a t t B$ site.

\begin{tabular}{|c|c|c|c|c|c|c|}
\hline $\begin{array}{l}\text { Insert } \\
\text { name }\end{array}$ & $\begin{array}{l}\text { Identity to } \\
\operatorname{attB}(\%)^{\mathrm{a}}\end{array}$ & $\begin{array}{c}\text { Identity to } \\
\text { minimal } a t t B(\%)^{b}\end{array}$ & $\begin{array}{l}\text { Identity with } \\
\text { attB-attP site }\end{array}$ & $\begin{array}{c}\text { Insertion } \\
\text { events }\end{array}$ & $\begin{array}{l}\text { Genome } \\
\text { position }\end{array}$ & Encoded protein \\
\hline Nt-1 & $26(36 \%)$ & $19(52 \%)$ & 5 & 1 & 731398 & MsrR family regulator \\
\hline $\mathrm{Nt}-2$ & $20(27 \%)$ & $14(38 \%)$ & 4 & 1 & 751814 & Disulfide bond forming protein \\
\hline $\mathrm{Nt}-3$ & $26(36 \%)$ & $17(47 \%)$ & 6 & 4 & 1066609 & Polyketide synthase \\
\hline $\mathrm{Nt}-4$ & $28(38 \%)$ & $19(52 \%)$ & 6 & 1 & 1505104 & Carboxymethylenebutenolidase \\
\hline Nt-5 & $30(41 \%)$ & $17(47 \%)$ & 3 & 1 & 1826014 & Non-coding region \\
\hline Nt-6 & $22(30 \%)$ & $13(36 \%)$ & 5 & 2 & 3715745 & Non-coding region \\
\hline $\mathrm{Nt}-7$ & $34(46 \%)$ & $20(55 \%)$ & 5 & 1 & 3939350 & FtsX-like permease \\
\hline $\mathrm{Nt}-8$ & $27(37 \%)$ & $15(41 \%)$ & 6 & 1 & 4126594 & Hypothetical protein \\
\hline Nt-9 & $25(34 \%)$ & $13(36 \%)$ & 3 & 1 & 4286641 & Hypothetical protein \\
\hline Nt-10 & $25(34 \%)$ & $17(47 \%)$ & 4 & 2 & 5761121 & Hypothetical protein \\
\hline Nt-11 & $20(27 \%)$ & $14(38 \%)$ & 4 & 1 & 7436287 & FadD15 fatty acid ligase \\
\hline $\mathrm{Nt}-12$ & $26(36 \%)$ & $16(44 \%)$ & 3 & 1 & 7530197 & Hypothetical protein \\
\hline Nt-13 & $25(34 \%)$ & $13(36 \%)$ & 3 & 1 & 7795264 & Putative deoxyribonuclease YcfH \\
\hline Nt-14 & $25(34 \%)$ & $19(52 \%)$ & 5 & 1 & 7823861 & Non-coding region \\
\hline Nt-15 & $25(34 \%)$ & $12(33 \%)$ & 4 & 1 & 8033744 & Telomeric repeat binding factor 2 \\
\hline Nt-16 & $35(48 \%)$ & $21(58 \%)$ & 6 & 1 & 8246408 & MNT1/THI5-like protein \\
\hline Nt-17 & $31(42 \%)$ & $16(44 \%)$ & 4 & 4 & 8620438 & Non-ribosomal peptide synthetase \\
\hline Nt-18 & $22(30 \%)$ & $14(38 \%)$ & 5 & 1 & 8670073 & Hypothetical protein \\
\hline Nt-19 & $23(31 \%)$ & $14(38 \%)$ & 4 & 1 & 8971553 & Phosphomevalonate kinase \\
\hline
\end{tabular}

$3 \quad{ }^{\text {a }}$ Number of identical nucleotide positions to the 73 bp canonical $\Phi$ BT1 attB site from $S$. coelicolor; ${ }^{\text {b }}$

4 Number of identical nucleotide positions to the $36 \mathrm{bp}$ minimal $\Phi$ BT1 attB site from S. coelicolor; ${ }^{\circ}$

5 Number of identical nucleotide positions to the $9 \mathrm{bp} S$. coelicolor attB- $\Phi$ BT1 attP recombination site

6 (Gregory et al. 2003). 


\section{Figure 1}

Genome and phylogenetic position of Nocardia terpenica AUSMD00012715

A) Genome map of $N$. terpenica 03-11436. Outer and inner blue and light blue lines represent CDS present on the forward and reverse strand, respectively. The innermost circle is a GC plot, showing variance in GC content across the genome. B) Concatenated ribosomal gene based phylogenetic tree showing the position of $N$. terpenica 03-11436 among 71 other sequenced Nocardia strains (including other sequenced as part of this study, marked by red circles). A maximum likelihood phylogeny was inferred under a $\mathrm{GTR}+\mathrm{F}+\mathrm{I}+\mathrm{G} 4$ model of nucleotide substitution, using as input an alignment of $2352 \mathrm{bp}$ concatenated nucleotide sequences for 5 ribosomal protein genes from 72 Nocardia. Asterisks indicate nodes with $<70 \%$ bootstrap support (1000 replicates).
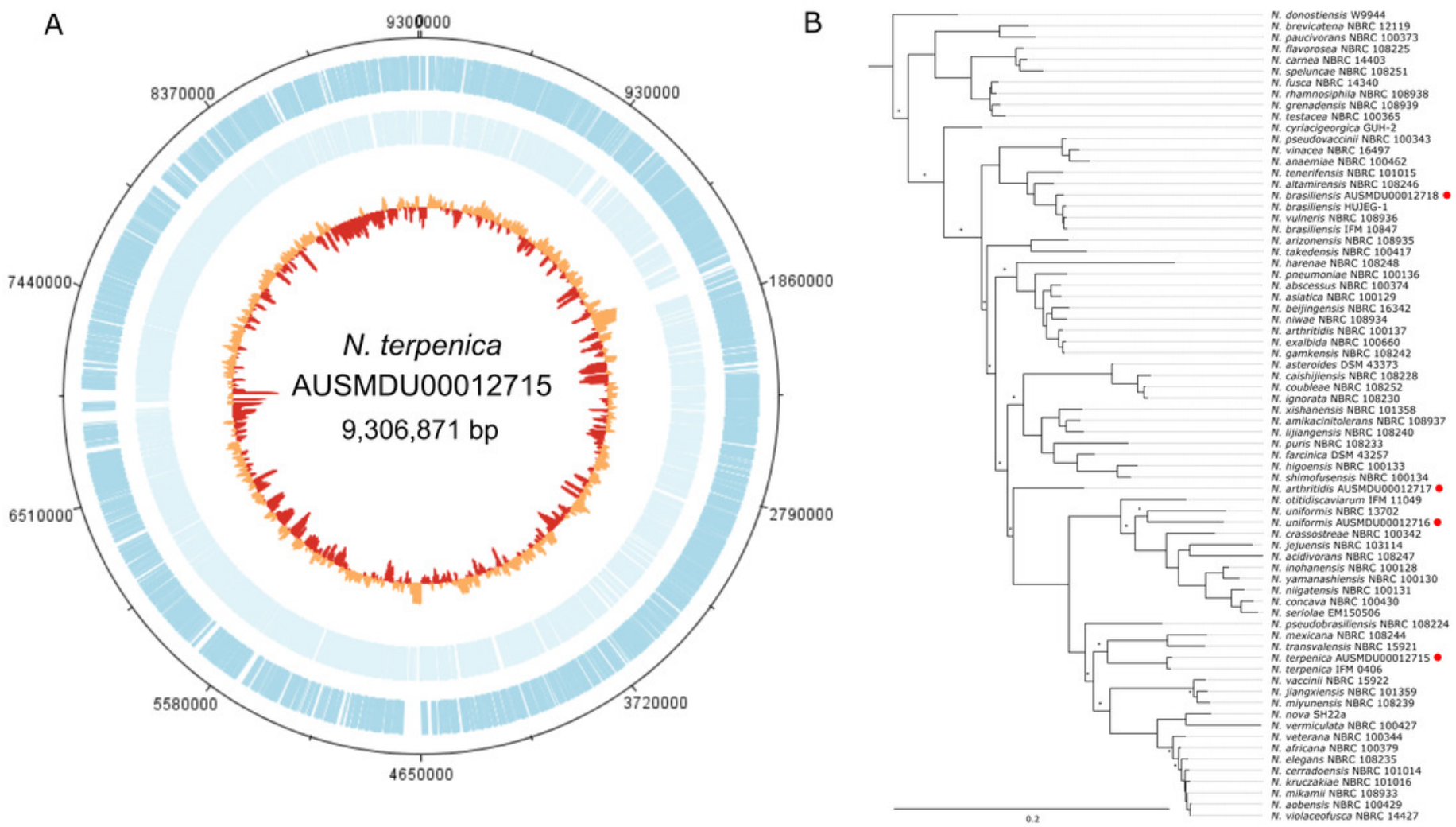


\section{Figure 2}

Pseudo-attB insertion sites in N. terpenica AUSMD00012715

A) Locations of individual pRT801 insertion sites mapped onto the N. terpenica AUSMDU00012715 genome. Percentage similarity to the minimal attB site of $S$. coelicolor is indicated by varying shades of red. B) Comparison of the most common $N$. terpenica pseudo-attB sites with the $73 \mathrm{~b}$ canonical attB sequence from $S$. coelicolor (a comparison of all sites can be found in Figure S1). Red nucleotides indicate identity to the canonical attB sequence. The common attB-attP core is boxed and the minimal attB sequence is underlined. The core GT dinucleotide is denoted by asterisks. C) Rarefaction analysis showing number of predicted sites in the $N$. terpenica AUSMDU00012715 genome.

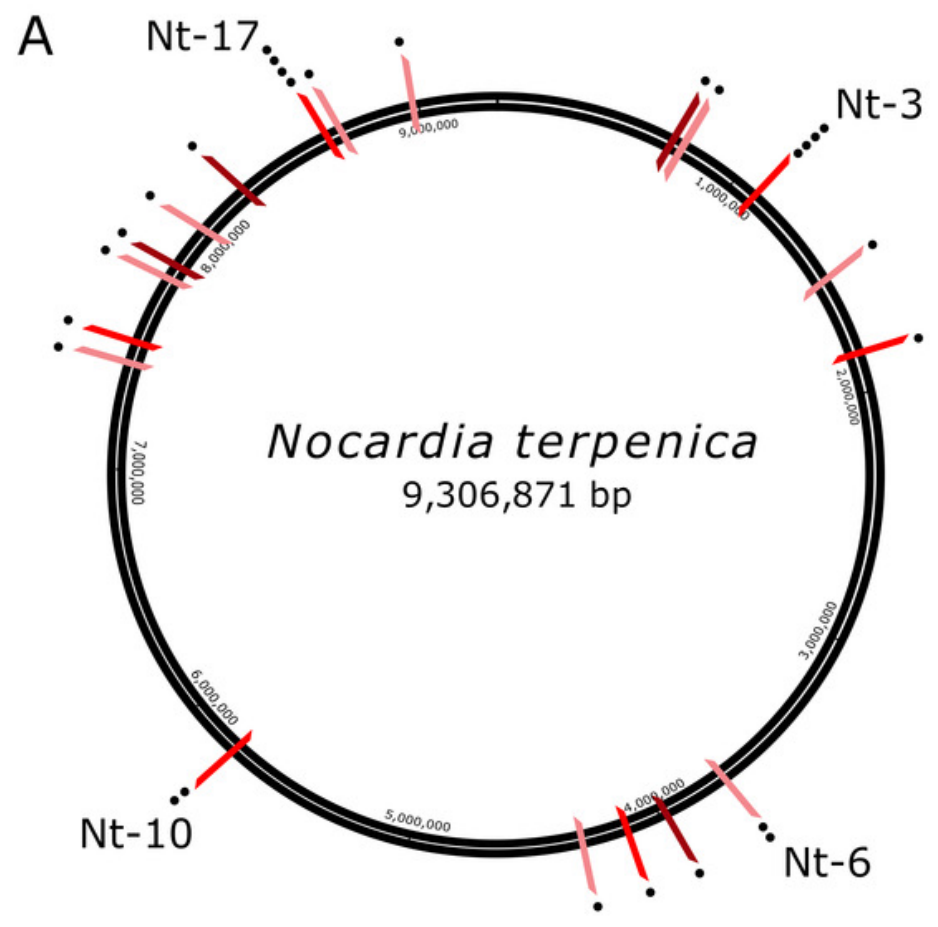

$\%$ identity to attB

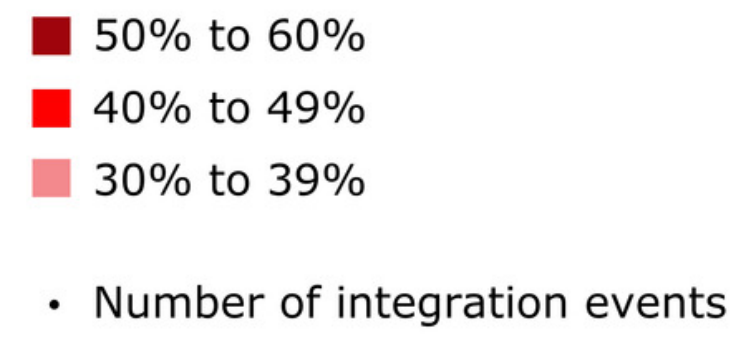

C

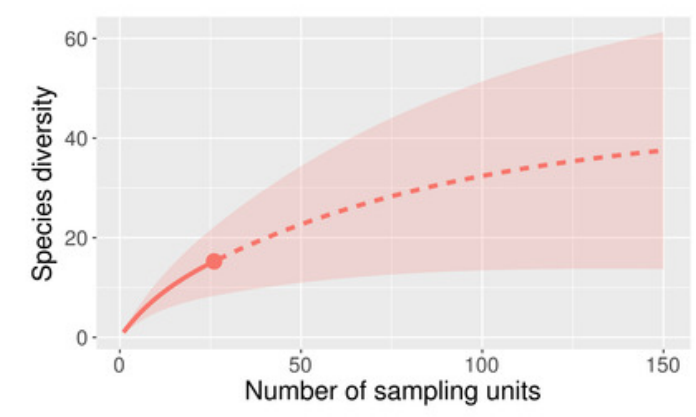

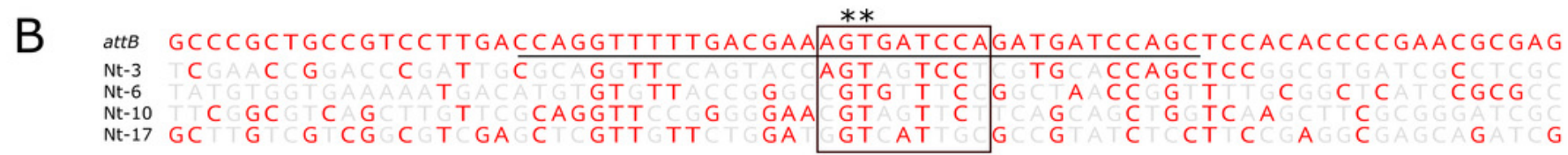


Figure 3

Comparison of attB sites in Streptomyces species

Comparison of S. coelicolor ФBT1 attB with those from S. albus J1074 (obtained from

GenBank), and Streptomyces strains sequenced as part of this study (S. albus J1074 (TS), S. coelicolor (TS) and S. cinnamonensis). The core GT site is in marked with asterisks, the minimal attB region is underlined and the $9 \mathrm{bp}$ overlap region is marked with a box. Red nucleotides are those that differ from the canonical attB sequence.

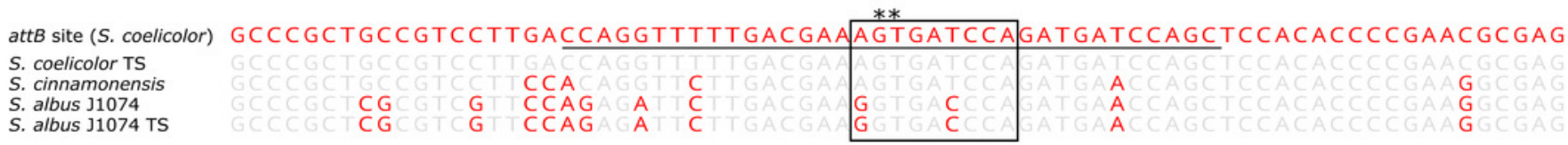




\section{Figure 4}

The pseudo-attB motif in Nocardia species and comparison of pseudo-attB sites with attP.

A) Alignment of attB sites with the pseudo-attB motif identified from Nocardia pseudo-attB insertion sites. Red nucleotides are those that are identical to the most common nucleotide at each position within the predicted motif. B) Alignment of $\Phi B T 1$ attP and attB sites. The canonical attP-attB sites from $\Phi \mathrm{BT} 1$ and $S$. coelicolor, respectively, are shown at the top, followed by an alignment of attP with the most common $N$. terpenica pseudo-attB sites, as well as those pseudo-attB sites that were most commonly seen from the other Nocardia species investigated in this study. The four predicted ZD binding motifs for ФBT1 Int on each attP and pseudo-attB half site are underlined, with the predicted RD binding site shown at the top. Residues that are identical in three of the four ZD motifs within each alignment are shown in red. Residues that are identical at three out of four positions within $-/+10$ of the RD binding region are also shown in red. The central GT dinucleotide is in red and labelled as position 0 . 
A

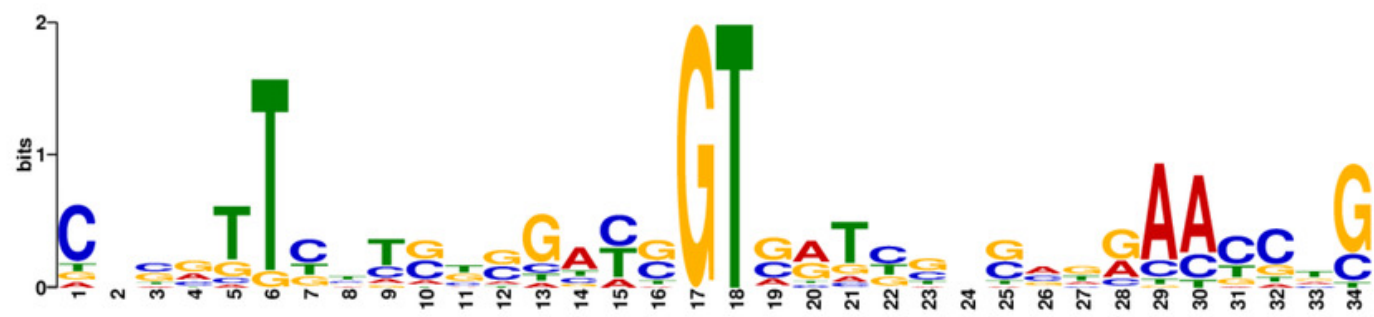

attB site (S. coelicolor)

S. lividans TK24

S. cinnamonensis

S. albus $\mathrm{J} 1074$

Nt-3

Nt-6

Nt-10

Nt-17

\begin{abstract}
C
\end{abstract}
CAGGTTTTTG

CAGGTTCTTG

TTCTTG $\mathrm{T}^{-}$

TGTGT

CAGGTTC

CTCGTT

$\mathrm{T}$
GA

GTGATC

$A G$

GA CCAG

$G A$
GTGATC

GAAAGTGATC AG

GA

CAGT

GGGCCAGTAGTC

GGA

$\mathrm{GT}$

AT

$G G A T G G T \subset A T \backslash G \subset G$

$\mathrm{G}^{\mathrm{G}}$

$\mathrm{G}$
B

attP site attB site

attP site

Nt-3

attP site

Nt-6

attP site

$\mathrm{Nt}-10$

attP site

$\mathrm{Nt}-17$

attP site

$\mathrm{Nb}-4$

attP site

$\mathrm{Na}-2$

attP site

$\mathrm{Nu}-1$
RD RD

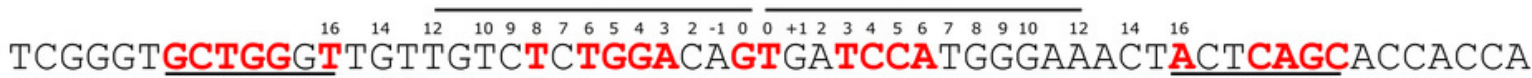
GCCGTCCTTGACCAGGTTTTTGACGAAAGTGATCCAGATGATCCA GCTCCACACCCCGA

TCGGGTGCTGGGTTGTTGTCTCTGGACAGTGATCCATGGGAAACTACTCAGCACCACCA GGACCCGATTGCGCAGGTTCCAGTACCAGTAGTCCTCGTGCACCAGCTCCGGCGTGATC TCGGGTGCTGGGTTGTTGTCTCTGGACAGTGATCCATGGGAAACTACTCAGCACCACCA TGAAAAATGACATGTGTGTTACCGGGCCGTGTTTCCGGCTAACCGGTTTTGCGGCTCAT TCGGGTGCTGGGTTGTTGTCTCTGGACAGTGATCCATGGGAAACTACTCAGCACCACCA TCAGCTTGTTCGCAGGTTCCGGGGGAACGTAGTTCTTCAGCAGCTGGTCAAGCTTCGCG

TCGGGTGCTGGGTTGTTGTCTCTGGACAGTGATCCATGGGAAACTACTCAGCACCACCA GTCGGCGTCGAGCTCGTTGTTCTGGATGGTCATTGCGCCGTATCTCCTTTCCGAGGCGA

TCGGGTGCTGGGTTGTTGTCTCTGGACAGTGATCCATGGGAAACTACTCAGCACCACCA

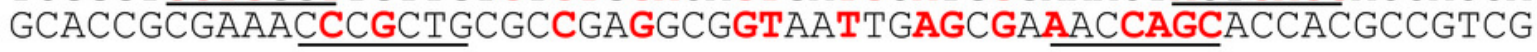

TCGGGTGCTGGGTTGTTGTCTCTGGACAGTGATCCATGGGAAACTACTCAGCACCACCA GCGAGGGCCGATGACATTTCTATGGCCGGTGCTGCTGCGAACTCT GCTGCGGCACACCA

TCGGGTGCTGGGTTGTTGTCTCTGGACAGTGATCCATGGGAAACTACTCAGCACCACCA GCAGCAGCGGAGCCGGTTTCACGCGATAGTCATCGCGCGGAACCGGCTCGTGTTGTCTC 